\title{
Erosion d'un lit déposé de sédiments cohésifs
}

\author{
Mathieu Mory, Ismael Piedra-Cueva ${ }^{1}$ et Philippe Larroudé \\ Laboratoire des Ecoulements Géophysiques et Industriels (UJF, INPG, CNRS) \\ BP 53, 38041 Grenoble Cédex 9
}

Une étude expérimentale de l'érosion d'un lit déposé de sédiments cohésifs par un courant stationnaire est présentée. La procédure expérimentale analyse l'érosion couche par couche d'un lit déposé lorsque la vitesse du courant est augmentée par paliers. Nos résultats expérimentaux, associés à une analyse des lois d'érosion proposées dans la littérature, conduisent à remettre en cause le concept d'érosion résiduelle. L'érosion varie linéairement avec l'excès de frottement, ce qui implique que l'érosion cesse lorsque le frottement est égal à la rigidité du lit. Une loi d'érosion adimensionnelle est établie dans le régime des faibles excès de frottement. Elle indique que le gradient de rigidité dans le lit est un des paramètres principaux régissant l'érosion d'un lit déposé.

\section{Introduction}

Une des principales applications des recherches sur le transport des sédiments fins cohésifs est l'ingénierie côtière. L'enjeu est notamment de permettre et maintenir la navigation dans les ports et les chenaux. Plus récemment, le rôle des sédiments dans le transport et la fixation des polluants est apparu. Ces exemples montrent l'intérêt de comprendre les processus d'érosion et de dépôt des sédiments cohésifs.

On présente les résultats d'une expérience dans laquelle le taux d'érosion d'un lit déposé et consolidé de sédiments cohésifs, par un courant stationnaire, a été mesuré. Il s'agit d'un système à deux couches. Le courant dans la couche supérieure applique une contrainte de frottement $\tau_{b}=\rho u_{*}^{2}$ sur le lití. La couche inférieure est le lit dont le comportement de type solide est caractérisé (entre autres) par la rigidité, traduite dans le cas présent par une contrainte de résistance à l'érosion $\tau_{\mathrm{s}}$. L'érosion du lit se produit lorsque $\tau_{b}>\tau_{s}\left(z_{b}\right)$. Pour un lit déposé et consolidé, la contrainte de résistance à l'érosion $\tau_{\mathrm{s}}$ croît avec la profondeur dans le lit et doit donc être connue à la surface du lit $z_{b}(t)$ pour déterminer si l'écoulement érode le lit. On définit le taux d'érosion selon

$$
\varepsilon=-\rho_{d}\left(z_{b}\right) L_{b} \frac{d z_{b}}{d t}=h L_{f} \frac{d \bar{C}}{d t} .
$$

La direction $z$ est verticale et orientée vers le haut. Les quantités $L_{f}$ et $L_{b}$ désignent respectivement la longueur du canal et la longueur du lit. Dans (1) le

1 adresse actuelle: Institute of Fluid Mechanics and Environmental Engineering (IMFIA), Faculty of Engineering, C.C. 30, Montevideo, Uruguay 
taux d'érosion est défini de façon équivalente, par conservation de la masse, en fonction de la variation du volume du lit ( $\rho_{d}$ est la densité sèche de sédiment dans le lit) ou de l'évolution temporelle de la concentration de sédiment en suspension moyennée sur la verticale $\bar{C}(t)$ (h est la profondeur de la couche d'eau).

Diverses études ont été réalisées sur le sujet. La nôtre est dans la continuité des travaux de Parchure et Mehta (1985). Lorsqu'un frottement constant est appliqué sur un lit déposé et consolidé, le taux d'érosion décroit dans le temps. Parchure et Mehta ont assimilé cette décroissance à une évolution exponentielle

$$
\varepsilon(t)=\varepsilon_{o} \exp (-\lambda t)
$$

qui implique que le taux d'érosion tend vers zéro à la fin d'un palier tandis que $\tau_{b}-\tau_{s}\left(z_{b}\right)$ suit la même évolution. Les expériences montrent que l'excès de cisaillement $\tau_{b}-\tau_{s}\left(z_{b}\right)$ est le paramètre principal gouvemant l'érosion. L'interprétation des variations du taux d'érosion en terme des variations de l'excès de frottement a conduit à la proposition de diverses lois d'érosion. Parchure et Mehta ont proposé la loi d'érosion suivante

$$
\varepsilon\left(z_{b}\right)=\varepsilon_{f} \exp \left[\alpha\left\{\tau_{b}-\tau_{s}\left(z_{b}\right)\right\}^{1 / 2}\right] \quad,
$$

dont il est important de faire remarquer qu'elle n'est pas adimensionnelle. Les lois (2) et (3) sont par ailleurs contradictoires, puisque (2) indique que l'érosion cesse à la fin d'un palier tandis que (3) implique qu'il subsiste une érosion non-nulle quand $\tau_{\mathrm{b}}-\tau_{\mathrm{s}}\left(\mathrm{z}_{\mathrm{b}}\right)=0 . \varepsilon_{\mathrm{f}}$ est appelé le taux d'érosion résiduelle.

On démontre en fait que la décroissance exponentielle avec le temps du taux d'érosion conduit à une loi d'érosion en fonction de l'excès de frottement de la forme

$$
\frac{\varepsilon\left(z_{b}\right)}{\varepsilon_{o}}=\frac{\tau_{b}-\tau_{s}\left(z_{b}\right)}{\tau_{b}-\tau_{s o}},
$$

si l'on suppose que la densité sèche en sédiment est constante dans la fine couche supérieure du lit érodée au cours d'un palier tandis que la résistance du lit à l'érosion varie linéairement. On définit $\tau_{s o}=\tau_{s}\left(z_{b}(t=0)\right)$. La loi linéaire d'érosion (4) fut proposée par Partheniades (1962).

Nous considérons dans cet article l'érosion en fin de palier afin de tester la pertinence de la notion d'érosion résiduelle. On cherche dans un premier temps à évaluer si la décroissance du taux d'érosion est exponentielle, en accord avec les observations de Parchure et Mehta. Dans un deuxième temps on analyse la loi d'érosion sous une forme adimensionnelle. 


\section{Procédure expérimentale}

Les expériences ont été réalisées dans le canal sédimentologique du Laboratoire d'Hydraulique de France, dont Piedra-Cueva, Mory \& Temperville (1997) (PMT) ont récemment présenté les potentialités pour l'étude du transport des sédiments cohésifs. La figure 1 montre un schéma de linstallation. Le courant est généré par une pompe constituée de disques verticaux dont la rotation entraine le fluide. La largeur du canal est $50 \mathrm{~cm}$. Toutes les expériences ont été réalisées avec une profondeur d'eau de $40 \mathrm{~cm}$. Le lit de vase était initialement déposé dans une fosse à sédiment de $8,2 \mathrm{~m}$ de longueur et $8 \mathrm{~cm}$ de profondeur, localisée dans la section droite du canal opposée à celle dans laquelle la pompe est située. Ce choix permet d'obtenir une érosion uniforme sur toute la surface du lit et élimine les fortes érosions dans les parties courbes du canal et au voisinage de la pompe.

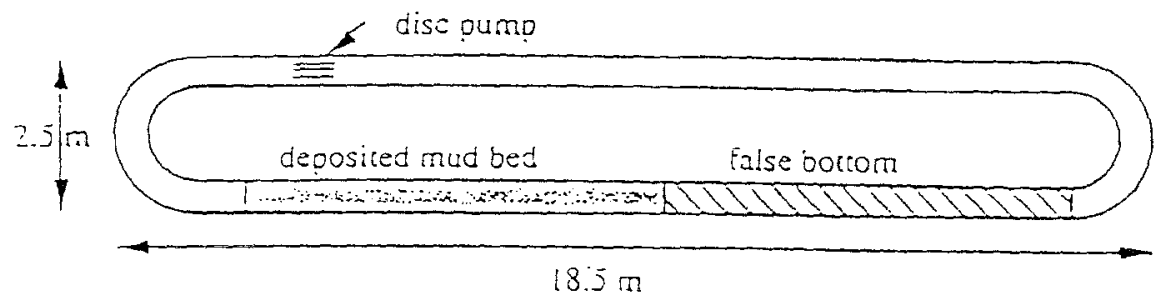

Figure 1 : Schéma de l'installation expérimentale.

Les expériences furent réalisées selon la méthode utilisée par Thorn \& Parsons (1979) et Parchure \& Mehta (1985). Le courant est augmenté par paliers dont la durée varie de $60 \mathrm{mn}$ à $180 \mathrm{mn}$. Une expérience consiste en général en 3 à 7 paliers. Le taux d'érosion décroît au cours de chaque palier jusqu'à atteindre une valeur très faible de sorte que le lit est érodé couche par couche au cours des différents paliers constituant une expérience.

Le taux d'érosion est déterminé à partir de la mesure continue de la concentration de sédiment en suspension qui est mesurée à trois niveaux dans le canal. La pompe à disques homogéneisant le sédiment sur la verticale, ces mesures donnent une estimation précise de la suspension moyenne sur la verticale.

\section{Détermination du taux d'érosion}

Le figure 2 montre l'évolution temporelle typique de la concentration de sédiment en suspension au cours d'un palier. Des oscillations sont visibles sur l'enregistrement expérimental. Elles apparaissent parce que le lit déposé ne couvre pas la totalité du fond du canal (voir PMT), et peuvent être filtrées par une opération de moyenne glissante. L'évolution moyenne du taux d'érosion a été estimée par l'ajustement d'une fonction de type exponentiel sur les enregistrements temporels 


$$
\bar{C}(t)=C_{\infty}-A \exp \{-\lambda t\}
$$

Les trois constantes $C_{\infty}, \lambda$ et $A$ sont déterminées par l'ajustement. $C_{\infty}$ est la concentration atteinte asymptotiquement à la fin du palier, qui diffère légèrement de la concentration $C_{m}$ mesurée à la fin du palier. Nous avons observé que la loi (5) ne donne jamais un ajustement satisfaisant sur toute la durée de l'enregistrement. Ceci est visible sur la figure 2 où la fonction interpolée (5) est superposée à l'enregistrement expérimental. Les constantes $C_{\infty}, \lambda$ et $A$ sont déterminées de manière à retenir le meilleur ajustement de la fonction (5) avec l'enregistrement expérimental sur la durée la plus longue possible à la fin du palier. L'ajustement est considéré significatif si $t_{m}>8 t_{1}$, où $t_{1}$ et $t_{m}$ désignent respectivement le début et la fin de la fraction de l'enregistrement sur lequel l'ajustement est retenu. Notre méthode d'analyse diffère fortement de celle employée par Parchure et Mehia (1985). De façon arbitraire, ces derniers ont analysé leurs données en considérant que le régime d'érosion résiduelle de floc était atteint en fin de palier lorsque le taux d'érosion était inférieur à $5 \%$ du taux d'érosion en début de palier. NYos ouservations nous conduisent à considérer que l'érosion de floc, si elle se produit, n'existe qu'à des taux d'érosion inférieurs à ceux que nous avons mesurés et en tout état de cause très inférieurs aux estimations de Parchure et Mehta.

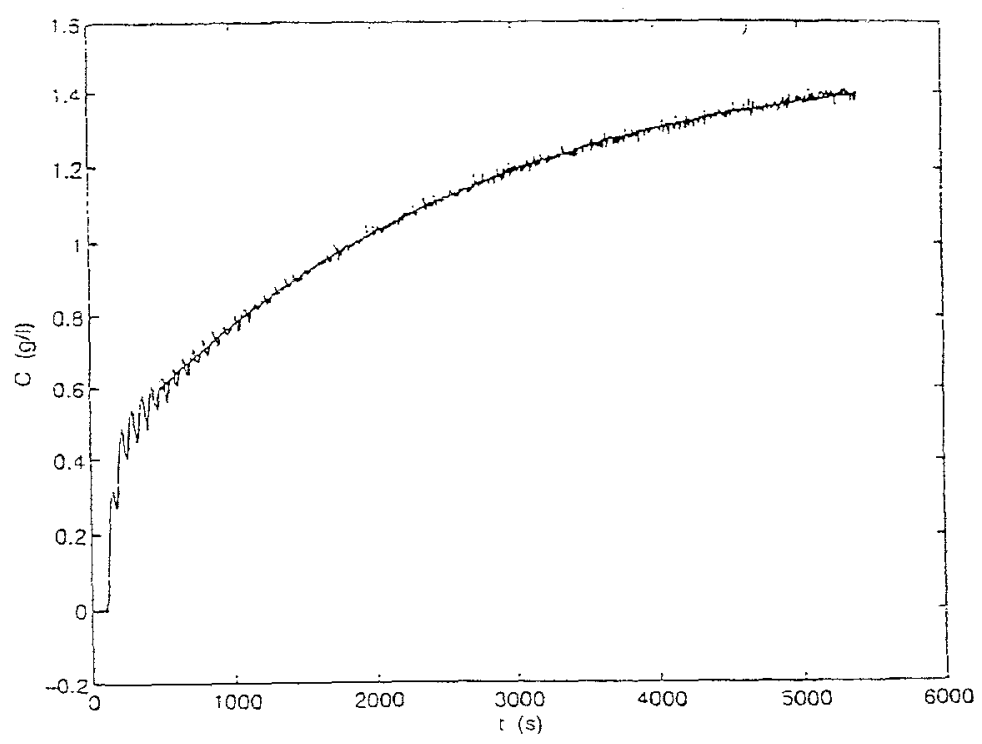

Figure 2: Variations temporelles de la concentration de sédiment en suspension au cours d'un palier. L'enregistrement expérimental (bruité et oscillatoire) est superposé avec la fonction de la forme (5) ajustée aux données expérimentales.

On déduit des variations temporelles du taux d'érosion en cours de palier, les variations du taux d'érosion en fonction des variations de l'excès de frottement, la décroissance exponentielle en fonction du temps du taux d'érosion (eq. 2) étant associée à une relation linéaire entre le taux d'érosion et l'excès de frottement (eq. 4). En introduisant le taux d'érosion non-dimensionnel $E=\varepsilon / \rho_{d} u_{*}$ et l'excès de frottement non-dimensionnel $T=\left(\tau_{b}-\tau_{s}\right) / \tau_{b} ;$ la relation (4) s'écrit

$$
E=K \quad T \text {, }
$$


où le coefficient de proportionnalité $K$ est déterminé pour chaque palier par

$$
K=\frac{\lambda C_{\infty} h}{\rho_{d} u_{*}} \frac{L_{f}}{L_{b}} \frac{\tau_{b}}{\tau_{b}-\tau_{s o}} .
$$

La constante $\mathrm{K}$ varie de façon importante d'un palier à l'autre comme le montre la figure 3 où les variations du taux d'érosion adimensionnel $E$ en fonction des variations de l'excès de frottement adimensionel $T$ sont tracées. Chaque droite représente un palier pour lequel la gamme de variation de $T$ est représentée. Les variations de pentes entre les différentes droites quantifient la variabilité du coefficient $\mathrm{K}$ entre les différents paliers.

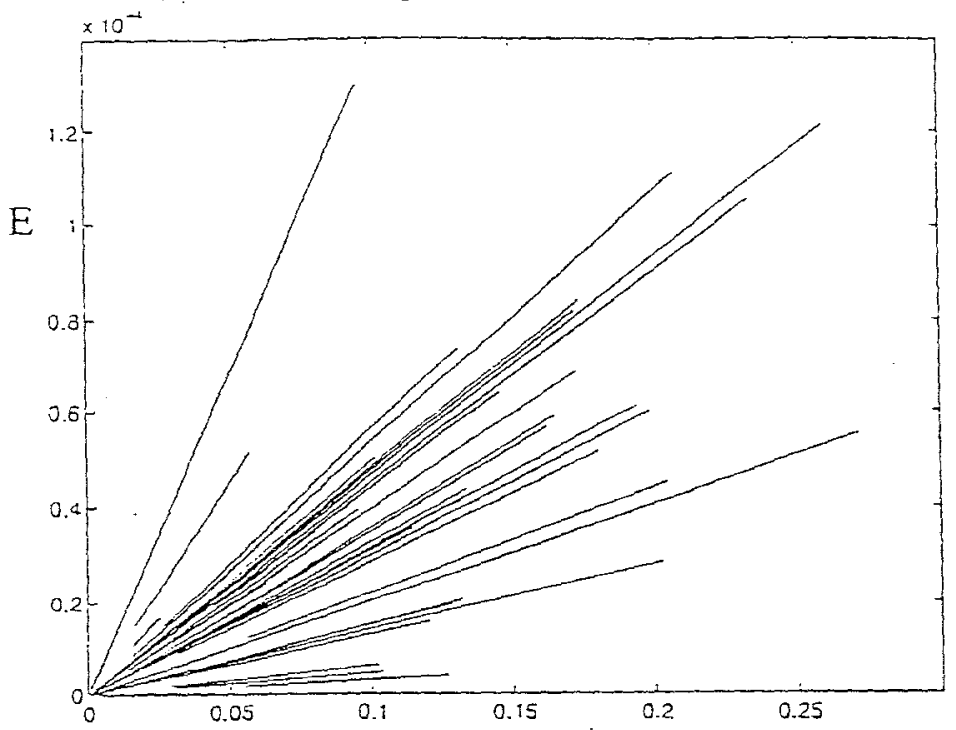

Figure 3: Variations du taux d'érosion en fonction de liexcès de frottement au cours de tous les paliers.

\section{Une analyse dimensionnelle des processus d'érosion}

Si l'excès de cisallement $\tau_{b}-\tau_{s}$ apparait clairement comme le paramètre principal gouvernant l'érosion, il n'est pas le seul. La formulation de lois d'érosion sous forme adimensionnelle n'est pas disponible. Nous examinons ci-dessous par une approche d'analyse dimensionnelle la dépendance du taux d'érosion avec les différents paramètres mécaniques, faisant l'hypothèse que les aspects physicochimiques et biologiques seront traduits à travers les paramètres mécaniques. L'érosion est supposée locale et dépend que des valeurs instantanées des différents paramètres. Nous prenons en compte les caractéristiques de la turbulence dans la couche d'eau et les propriétés mécaniques dans le lit. La dépendance du taux d'érosion est donc la suivante

$$
\varepsilon(t)=\varepsilon\left\{g, \rho, \mu, h, \tau_{b}, \rho_{d}, \mu_{d}, H, \tau_{s}\right\}
$$

La dynamique dans la couche d'eau fait intervenir la masse volumique de l'eau $\rho$, la viscosité dynamique $\mu$, l'épaisseur de la couche d'eau h et la contrainte de frottement sur le fond $\tau_{b}$. La résistance à l'érosion à la surface du lit $\tau_{s}$, la masse 
volumique sèche de sédiment $\rho_{d}$ et la viscosité dynamique $\mu_{d}$ caractérisent les propriétés du lit. La contrainte de résistance à l'érosion à la surface du lit $\tau_{\mathrm{s}}$ diffère de la rigidité $\tau_{y}$ telle qu'elle est définie pour un matériau de Bingham et mesurée dans un rhéomètre. Les mesures indiquent que la résistance à l'érosion est inférieure à la rigidité (Migniot 1989). H est l'épaisseur de la couche de surface dont la résistance à l'érosion est inférieure à la contrainte de frottement. Comme $\tau_{b}-\tau_{s}>0$, cette couche est érodée continûment au cours d'un palier. $\tau_{s}\left(z_{b}(t)\right)$ et $H(t)$ sont des quantités variables dans le temps.

L'analyse dimensionnelle montre que le taux d'érosion adimensionnel a la dépendance suivante

$$
E=\frac{\varepsilon(t)}{\rho_{d} u_{*}}=F\left\{\begin{array}{l}
T=\frac{\tau_{b}-\tau_{s}}{\tau_{b}}, R i=\frac{\rho_{d} g h}{\tau_{b}}, I=\frac{\tau_{b} H}{\left(\tau_{b}-\tau_{s}\right) h}, \\
\operatorname{Re}_{e}=\frac{h \sqrt{\rho \tau_{b}}}{\mu}, R=\frac{\rho_{d} \tau_{b}^{3} H^{2}}{\mu_{d}^{2}\left(\tau_{b}-\tau_{s}\right)^{2}}
\end{array}\right\}
$$

$u_{*}=\sqrt{\tau_{b} / \rho}$ est la vitesse de frottement à la surface du lit. A côté de l'excès de frottement $\mathrm{T}$ et du nombre de Richardson $\mathrm{Ri}$ (comparant la gravité au gradient de la contrainte de la couche fluide), qui sont classiques, la quantité I compare le gradient des contraintes dans la couche d'eau et dans le lit. Le rôle de cette quantité n'a pas été mentionné par le passé, à notre connaissance. Les deux dernières quantités $\mathrm{Re}_{\mathrm{e}}$ et $\mathrm{R}$ quantifient les effets dissipatifs dans la couche d'eau et le lit, respectivement. La formulation de $\mathrm{R}$ est un peu particulière. Sur le plan physique, elle s'appuie sur un modèle de type Bingham. Dans le cas particulier de notre expérience, l'expression des quantités $\mathrm{Ri}, \mathrm{I}, \mathrm{Re}_{\mathrm{e}}$ et $\mathrm{R}$ ont été choisies de manière à garder ces quantités constantes au cours d'un palier. La seule quantité variable au cours d'un palier est T. La comparaison de (6) et (9) indique que la loi d'érosion peut être analysée à partir de la relation de dépendance suivante pour le coefficient $\mathrm{K}$

$$
E=K\left(I, R i, \operatorname{Re}_{e}, R\right) T
$$

Les variations de $\mathrm{K}$ avec les différentes quantités adimensionnelles ne sont pas toutes accessibles de nos expériences. L'écoulement étant pleinement turbulent dans la couche d'eau, l'effet du nombre Reynolds $R_{e} n^{\prime}$ 'est pas significatif. En l'absence de données sur la rhéologie dans le lit, le nombre Reynolds dans le lit $\mathrm{R}$ est inconnu dans nos expériences et la dépendance de $K$ avec $R$ est donc indéterminée. La densité sèche dans le lit est forte et le nombre de Richardson est grand. Pour l'essentiel de nos expériences, Ri était compris entre 1200-2000. Cet intervalle est trop faible pour mettre en évidence une dépendance de $\mathrm{K}$ avec $\mathrm{Ri}$. En revanche, la quantité I varie d'un palier à l'autre entre 0.01 et 0.30 . La figure 4 présente les variations du coefficient de proportionnalité $\mathrm{K}$ avec I. La variabilité des résultats est importante. La part dans cette variabilité liée à la procédure d'ajustement utilisée pour déterminer le taux d'érosion a été estimée et est évaluée sur la figure 4 par les barres d'erreur. Par ailleurs les symboles noircis indiquent les cas où la concentration asympotique $C_{\infty}$ est inférieure à $0.35 \mathrm{~g} / \mathrm{l}$. L'incertitude 
expérimentale associée à la précision des turbidimètres peut expliquer la large variabilité pour les faibles valeurs de I. En dépit de ces remarques, la croissance de $K$ avec $I$ est visible. Elle indique que l'érosion d'un lit déposé dépend du gradient de rigidité dans le lit Le taux d'érosion diminue lorsque le gradient de rigidité augmente à la surface du lit.

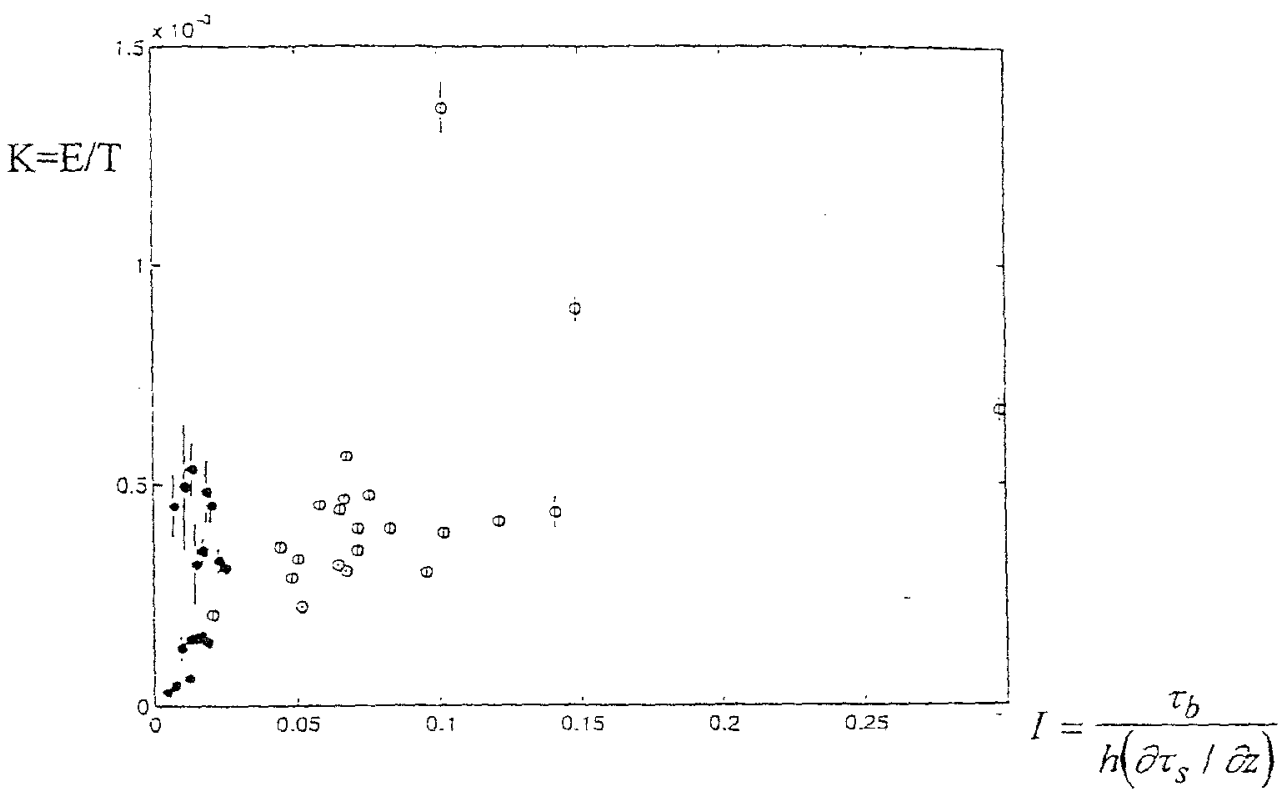

Figure 4 : Variation du coefficient de proportionnalité entre taux d'érosion et excès de cisaillement avec le gradient de rigidité dans le lit.

\section{Conclusion}

Des expériences ont été réalisées sur l'érosion, d'un lit déposé et consolidé de sédiments cohésifs, par un courant. Le sujet n'est pas nouveau mais notre travail montre que la méthode d'analyse des données explique certaines contradictions dans la littérature. Une forme de la loi d'érosion a été analysée, à notre connaissance pour la fois première fois de façon adimensionnelle. Notre travail remet en cause le concept d'érosion résiduelle de floc. Si un tel phénomène existe, il est très faible. L'observation indique que l'érosion cesse lorsque la contrainte de frottement est égale à la résistance à l'érosion. A la fin de chaque palier, une décroissance exponentielle avec le temps du taux d'érosion est observée. Cette loi temporelle implique une relation linéaire entre le taux d'érosion et l'excès de frottement. Un telle loi fut proposée par Partheniades (1962). La formulation, exprimée dans notre travail sous une forme adimensionnelle, confirme que l'excès de cisaillement est le paramètre principal régissant l'érosion. La loi d'érosion fait par ailleurs apparaitre le gradient à la surface du lit de la contrainte de résistance à l'érosion dans le dimensionnement de la loi d'érosion. Les résultats présentés dans cette article ne concement que l'érosion produite en fin de palier, cas pour lequel l'excès de cisaillement est faible (inférieur à 0,25 en valeur adimensionnelle, dans tous les cas). La plus grande inconnue des expériences est l'indétermination du comportement rhéologique dans le lit, qui intervient dans le dimensionnement de 
l'érosion. Toutefois, aux faibles valeurs de cisaillement produits dans le lit pour les valeurs de l'excès de frottement considérées ici, les variations temporelles dans le lit liées à la rhéologie et aux phénomènes de thixothropie peuvent être vraisemblablement écartées.

\section{Remerciements}

Ce travail a été réalisé dans le cadre des projets MAST-2 G8M Coastal Morphodynamics (contract MAS2-CT-92-0027) et MAST-3 COSINUS (contract MAS3-CT-97-0082), financés par la Commission des Communautées Européennes, Direction Générales des Sciences. Le Laboratoire d'Hydraulique de France, le gouvernement français, le CONICYT et la Faculté d'Ingénierie de l'Université de la République d'Uruguay sont également remerciés.

\section{Références}

MIGNIOT C. 1989 Tassement et rhéologie des vases, deuxième partie. La Houille Blanche, 2, 95-111.

PARCHURE TM. \& MEHTA A.J. 1985 Erosion of soft cohesive sediments deposits. J. Hydraulic Engineering, 111(10), 1308-1326.

PARTHENIADES E. 1965 Erosion and deposition of cohesive soils. J. Hydraulics Division, ASCE, 91(HY1), 105-139.

PIEDRA-CUEVA I., MORY M. \& TEMPERVILLE A. 1997 A race-track recirculating flume for cohesive sediment research. J. Hydraulic Research, 35(3), 377-396.

THORN MF.C. \& PARSONS J.G. 1979 Properties of Belawan muds. Technical Report EX 880, Hydraulics Research Station, Wallingford. 